\title{
Haze Detection and Removal in Remotely Sensed Multispectral Imagery
}

\author{
Aliaksei Makarau, Rudolf Richter, Rupert Müller, and Peter Reinartz
}

\begin{abstract}
Haze degrades optical data and reduces the accuracy of data interpretation. Haze detection and removal is a challenging and important task for optical multispectral data correction. This paper presents an empirical and automatic method for inhomogeneous haze detection and removal in medium- and high-resolution satellite optical multispectral images. The dark-object subtraction method is further developed to calculate a haze thickness map, allowing a spectrally consistent haze removal on calibrated and uncalibrated satellite multispectral data. Rare scenes with a uniform and highly reflecting landcover result in limitations of the method. Evaluation on hazy multispectral data (Landsat 8 OLI and WorldView-2) and a comparison to haze-free reference data illustrate the spectral consistency after haze removal.
\end{abstract}

Index Terms-Haze removal, Landsat 8 OLI, spectral consistency, WorldView-2.

\section{INTRODUCTION}

$\mathbf{H}$ AZINESS is a common artifact in optical remotely sensed data originating from fractions of water vapor, ice, fog, sand, dust, smoke, or other small particles in the atmosphere. Haze transparency leaves an opportunity for optical imagery restoration; nevertheless, for an inhomogeneous and structured haziness (see the example shown in Fig. 1), a precise detection is necessary, and a haze thickness map (HTM) has to be calculated. An efficient method for multi- and hyperspectral remotely sensed optical data dehazing handling all types of haziness and haze thickness is still a challenge.

Richter [1] developed a dehazing method accurately dealing with haze transition (haze-nonhaze) regions. Clear, hazy, and cloud regions are separated and stored. A haze boundary region is introduced to generate a smoother transition from haze to clear areas. The algorithm matches the histogram of the haze areas to the histogram of the clear part leading to dehazing.

Moro et al. [2] presented a framework to reduce haze and calculate some vegetation indices. Haze removal is performed based on the haze optimized transform (HOT; see original work of [3]) technique. The improved HOT consists of the following: determination of a man-made feature mask, masking of estimated haze for water bodies and man-made features, interpolation of masked-out areas, and final haze subtraction from the image. Dark-object subtraction (DOS) is then performed

Manuscript received August 17, 2013; revised October 2, 2013 and October 31, 2013; accepted November 21, 2013.

The authors are with the German Aerospace Center (DLR), 82234 Wessling, Germany (e-mail: aliaksei.makarau@dlr.de).

Color versions of one or more of the figures in this paper are available online at http://ieeexplore.ieee.org.

Digital Object Identifier 10.1109/TGRS.2013.2293662 on the dehazed scene in order to estimate approximate soil reflectances necessary to calculate the vegetation indices.

Liu et al. [4] developed a technique to remove spatially varying haze contamination, comprising three steps: haze detection, haze perfection, and haze removal. The background suppressed haze thickness index (BSHTI) is used to indicate relative haze thickness. The virtual cloud point method based on BSHTI is used in haze removal. Human intervention is necessary to outline thick haze and clear regions. Here, 76 paired (dehazed and haze-free) regions were compared, resulting in a correlation coefficient higher than 0.7 .

He et al. [5] proposed an efficient haze removal and depth map estimation method for outdoor colored RGB images. The method is based on dark channel prior calculation (a search of local patches in the image containing pixels with very low intensity). The hazy image dark channel values are mainly contributed by the airlight (path radiance), and the dark pixels can provide an estimation of the haze transmission. A haze imaging model allows us to recover a high-quality haze-free image and a corresponding depth map.

Chavez [6], [7] proposed an improvement for DOS technique to correct optical data for atmospheric scattering. The method assumes to find a pixel or several pixels with a very low reflectance. Due to the haziness in the scene, these pixels are not completely dark, and the digital numbers (DNs) in the pixels are nonzero. The values of the DNs are assumed to be the haze thickness in the image. Assuming a constant haze over the image, a subtraction of the DN value from the whole image allows us to perform dehazing.

In this paper, we present a further development of the DOS technique by Chavez [6]. Instead of searching only several dark objects in the whole scene, it is proposed to search dark objects locally in the whole image. A local search of the dark objects allows us to construct an HTM. Assuming an additive model of the haze influence, a subtraction of the HTM from a hazy image allows us to restore the haze-free signal at the sensor. The dehazed data can be later employed for atmospheric correction [8] and for further processing like landcover classification [9][11] or change detection [12].

This paper is organized as follows. In Section II, the new haze removal algorithm is presented. The method of the HTM calculation as well as a correction for large highly reflective objects is given. Haze thickness estimation per band is developed, allowing multispectral image dehazing. Section III contains a description of hazy and haze-free data, data properties, and examples of dehazed data evaluation. The conclusion and possible further developments of the method are given at the end of this paper. 


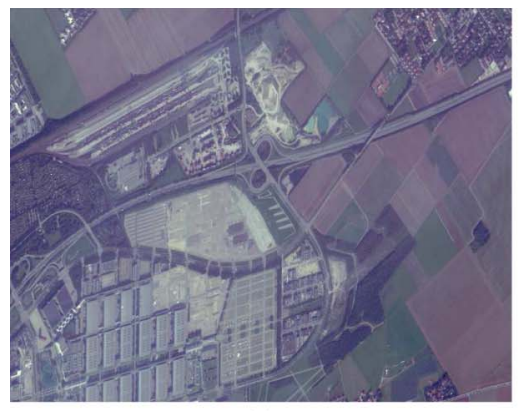

(a)

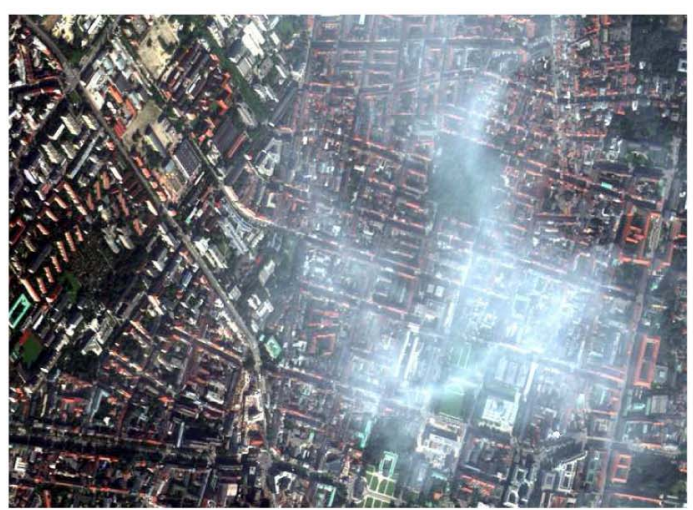

(c)

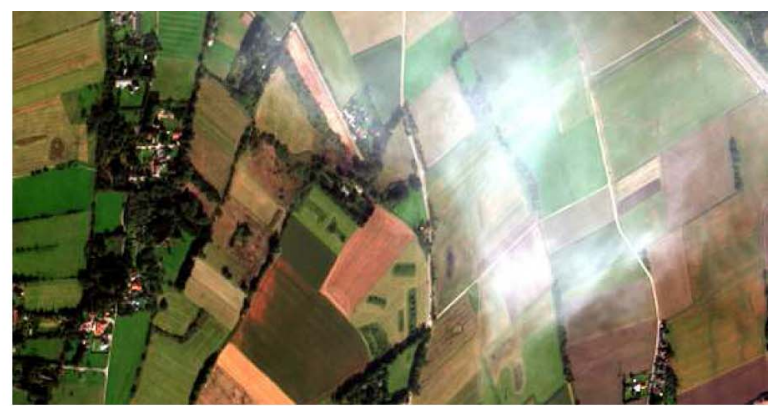

(b)

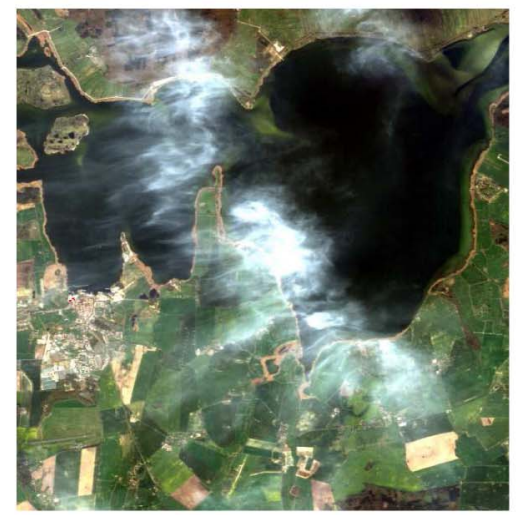

(d)

Fig. 1. Typical types of haziness in optical imagery. (a) Uniform haze (WorldView-2). (b) Moderately thick haze (WorldView-2). (c) Moderately thin haze (WorldView-2). (d) Thick structured haze (AVNIR).

\section{Proposed Method for Haze Removal}

\section{A. Haze Model}

Small field-of-view optical systems are employed to acquire medium, high, or very high spatial resolution multispectral data. Then, the distance from any object in the scene to the sensor can be assumed as constant. Our simplified model (assumption is verified in the experiments) can be described as follows (see the additive model by Chavez [6]):

$$
L^{\text {sensor }}=L_{0}+H R
$$

where $L^{\text {sensor }}$ is the acquired radiance, $L_{0}$ is the sum of the path radiance and the surface reflected radiance, and $H R$ is the haze contribution.

Assuming a linear nature of the DN to radiance conversion, the equation is adapted to band $\mathrm{DN}$

$$
\mathrm{DN}_{i}^{\text {sensor }}(x, y)=\mathrm{DN}_{i}(x, y)+H R_{i}^{\prime}(x, y)
$$

where $(x, y)$ are the coordinates of a pixel in an optical image, $i$ is the band number, $\mathrm{DN}_{i}^{\text {sensor }}(x, y)$ is the acquired DN, and $\mathrm{DN}_{i}(x, y)$ is the DN without the influence of haze. The term $H R_{i}^{\prime}(x, y)$ is dependent on the haze thickness and can be defined as $\operatorname{HTM~}_{\operatorname{HTM}}(x, y)$.

\section{B. HTM}

Since the HTM is employed as an additive term (2), a subtraction of the HTM from an optical image allows us to restore the haze-free image. The task is to calculate $\operatorname{HTM}_{i}(x, y)$ for each band $i$.

Haze thickness is usually variable over a scene [e.g., Fig. 1(b)-(d)], and the HTM has to be calculated by searching dark pixels (shaded objects [6]) over all of the image. A medium to small ground sampling distance (GSD; 30-2 m) of a sensor allows us to record shadows from objects. The shading can be caused by a varying relief in the scene, natural or manmade objects. These pixels have a very low $\mathrm{DN}$ value (small ground reflectance+path radiance), and with the presence of haze, the recorded DN value is also nonzero and higher than the corresponding haze-free contribution. These nonzero DN values can be employed to estimate the HTM of the image. It should be noted that the HTM includes the thickness of the haze and the aerosol thickness. The compensation for the subtracted clear scene aerosol thickness is given in the dehazing section (Section II-E).

The dark pixels are searched using a local nonoverlapping window $w$ (i.e., area $w \times w$ pixels). Pixelwise search for dark pixels can result in an occasional selection of pixels over nonshaded or bright objects, and these pixels cannot be used to estimate haze thickness (the HTM will contain errors). The window is employed to increase the chance to locate dark pixels over shaded regions. The other pixels in the window are assumed to have the same haze thickness as the thickness estimated in the pixel with the minimal value. A larger window allows easier detection of dark pixels, but the border as well as the structure (density in each pixel or local area) of haze can be smoothed, leading to an underestimation of the HTM (Fig. 2). A smaller window allows better estimation of haze 


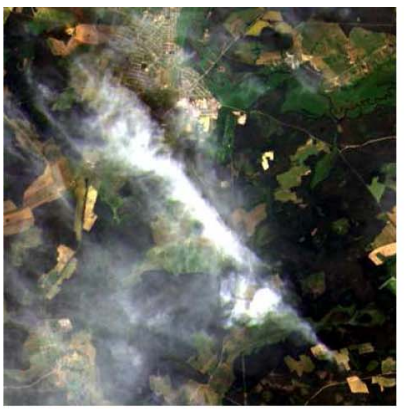

(a)

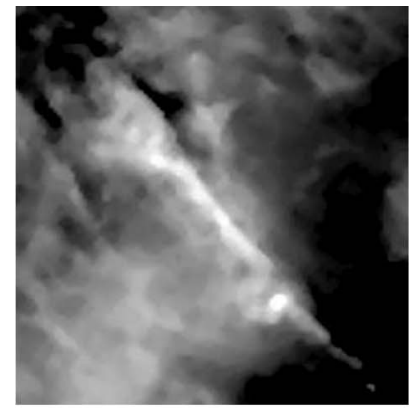

(b)

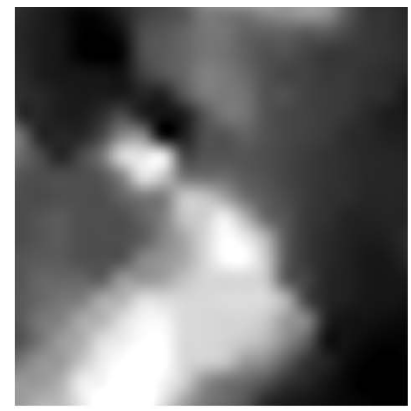

(c)

Fig. 2. HTM preciseness dependence on the nonoverlapping window size. (a) Example of an image (Landsat 8 OLI, RGB true color combination) with inhomogeneous haze (thick in the middle but moderately thin over all of the subimage). (b) HTM calculated using the window size $w=5$. (c) HTM calculated using the window size $w=21$

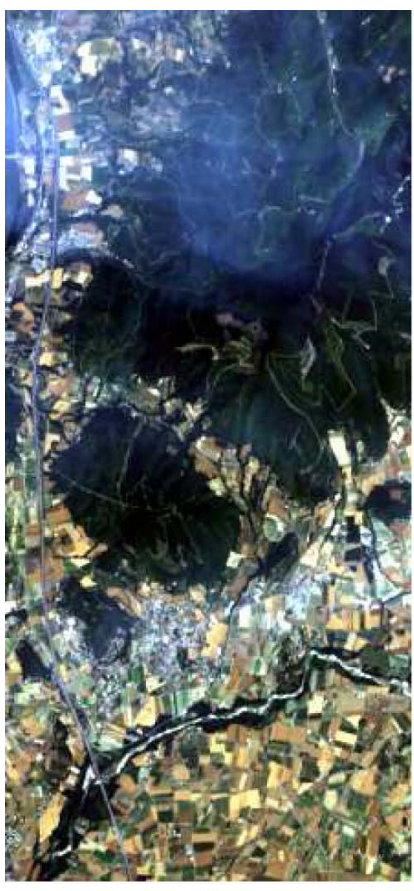

(a)

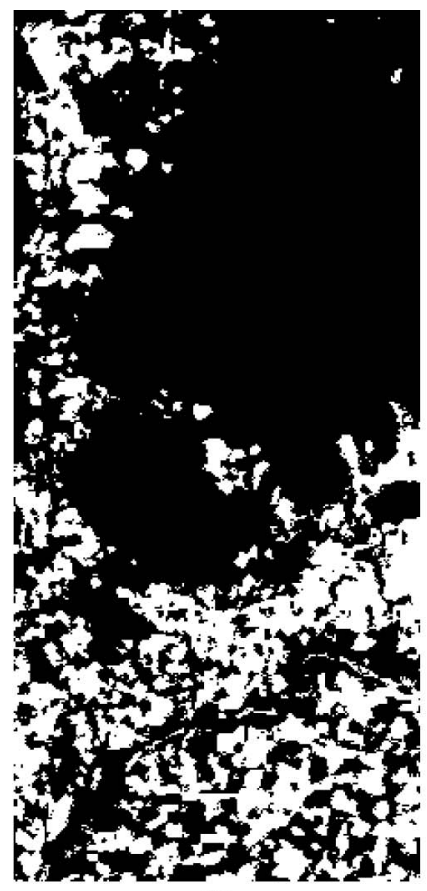

(b)

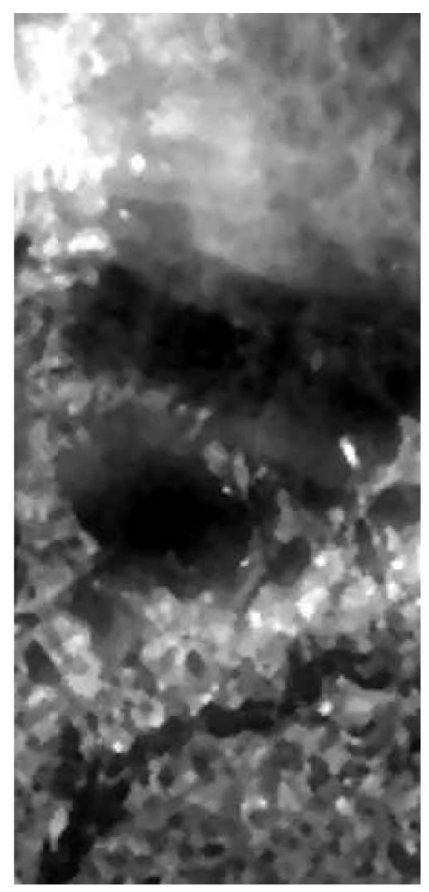

(c)

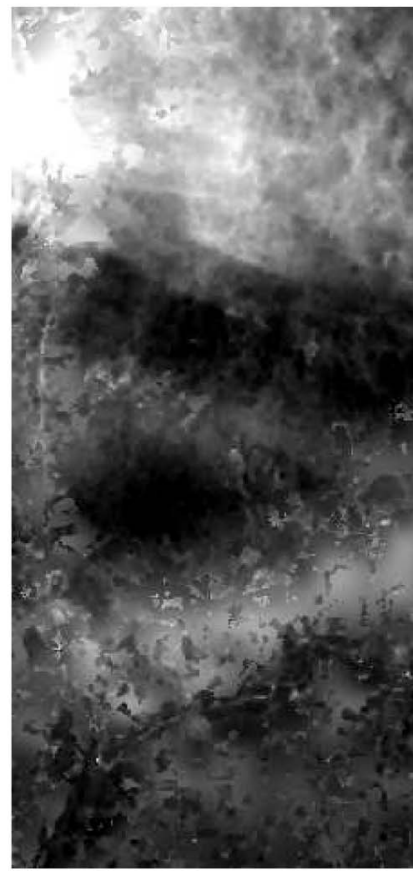

(d)

Fig. 3. Example of the HTM calculation for an area containing large objects with moderately high reflectance (soils). The HTM is corrected for the regions with the bright objects. (a) Landsat 8 OLI RGB true color band combination (haze noticeable at the upper part of the image). (b) Mask for large bright objects. (c) HTM (large bright objects can be confused with haze). (d) Corrected HTM (the bright object pixels are interpolated by triangulation).

structure together with a possibility that outside the haze pure dark pixels are not found (and the pixels with a moderately high intensity are selected). The size of the window is set according to the GSD of the sensor: the smaller the GSD, the smaller size of the window can be set, and a more precise HTM can be estimated. Usually, the size of the window is constant for a selected sensor. By the experimental analysis, it is found that the minimum window size should be three times higher than the sensor GSD, e.g., Landsat 8 OLI (30-m GSD for multispectral data) should have a minimum window size of $90 \mathrm{~m} \times 90 \mathrm{~m}$, and WorldView-2 (2-m GSD for multispectral data) should have a minimum window size of $6 \mathrm{~m} \times 6 \mathrm{~m}$, respectively. The dark pixels selected using the window (the size is $w \times w$ ) are stored in a matrix with the size $w$ times smaller than the original hazy image. Then, the matrix is smoothed by a median filter (usually $3 \times 3$ ) and interpolated (cubic interpolation) up to the size of the original band. Therefore, the possible haze overestimation is suppressed.

The presence of large bright objects can make the selection of dark pixels difficult using a small window (see Fig. 3). Such objects can be agricultural fields, regions with bright sand, uniform snow cover, etc. These objects are labeled by a segmentation method (in this work, a mean-shift segmentation is employed [13], but any other segmentation methods can be also used). The HTM values in the regions of bright objects are interpolated using a triangulation. In an extreme case having a uniform landcover in the whole scene with a high reflectance (e.g., snow), the HTM might be impossible to estimate. Usually, such cases are rare in remotely sensed data and not of interest for the use of this methodology.

The HTM calculation depends on the employed spectral band for the dark pixel search and the size of the nonoverlapping 


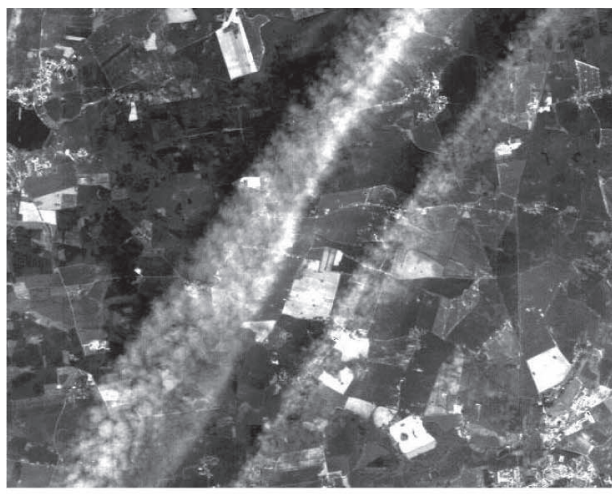

(a)

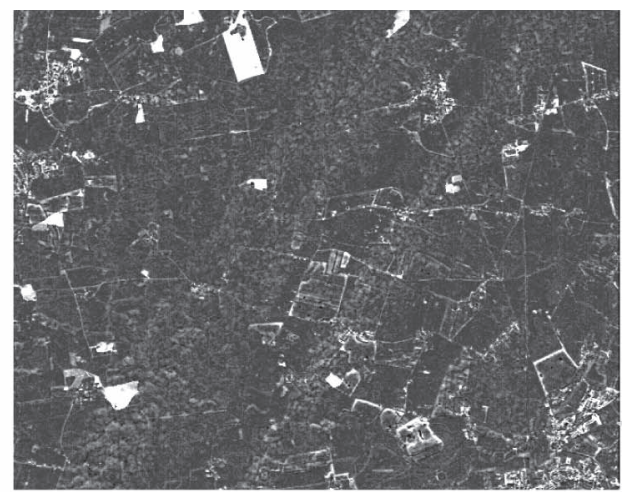

(b)

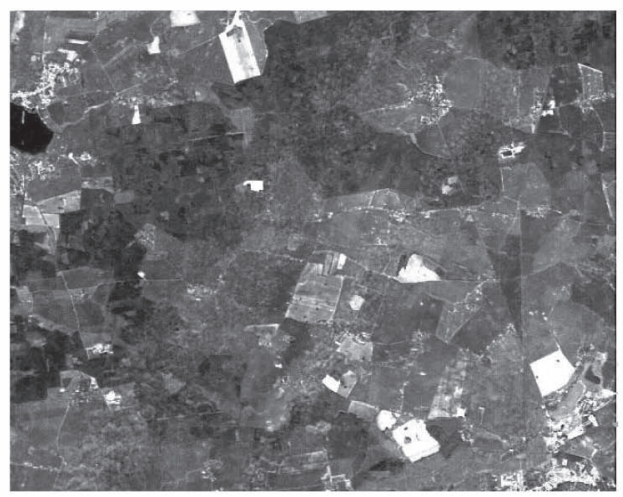

(c)

Fig. 4. AVNIR-2 data dehazing example (subset). The employment of band $1(0.42-0.50 \mu \mathrm{m})$ for the HTM calculation can lead to overdehazing in the same band. (a) Original band 1. (b) Dehazed band 1 (overdehazing is notable). (c) Dehazed band 1 (HTM was calculated using a new band produced by extrapolation of AVNIR-2 band 1 and band 2).

window

$$
\operatorname{HTM}(x, y)=D A R K_{\_} P I X \_S E A R C H\left(\operatorname{Band}_{b}(x, y), w\right)
$$

where $\operatorname{Band}_{b}(x, y)$ is the employed image (usually a blue band), $w$ is the size of the nonoverlapping window, and $\operatorname{HTM}(x, y)$ is the HTM (unitless). In our experiments, $w$ usually takes values ranging from 3 to 9 . With $w=3$, reasonable results on medium- and high-resolution data (Landsat 7, Landsat 8 OLI, Advanced Visible and Near Infrared Radiometer (AVNIR), AVIRIS, WorldView-2, and RapidEye) were achieved.

It is necessary to label hazy and haze-free regions. The information in haze-free regions is further employed in the dehazing and compensating of clear scene aerosol. In order to find haze-free regions, the haze mask is created. The haze mask is calculated by the generation of an additional HTM with moderately large window size $(\mathrm{GSD} * 20, w=21)$ and a thresholding of the HTM. The HTM generated with the large window size is used only to label hazy and haze-free regions.

The coefficient of 20 is estimated experimentally. The threshold value is equal to the mean value (water area under haze is segmented with the employment of the near infrared band and excluded), and reasonable results were achieved for all processed data. The haze mask as well as the water mask does not necessarily have to be precise, and a mislabeling of objects in the scene does not influence dehazing (the haze mask is used only to calculate a relative haze thickness coefficient, and this is robust to mislabeled outliers). The employment of such large windows allows us to find dark pixels and estimate the HTM irrespectively of large bright objects.

In multispectral data, the search of dark pixels should be performed in a band with a minimal ground reflectance and a maximal attenuation by haze. A spectral band in the blue spectral region $(0.37-0.49 \mu \mathrm{m})$ is most suitable for this purpose. Spectral bands in the red or near infrared spectral regions exhibit higher ground reflectance for land and are less influenced by haziness; therefore, they are not suitable in detecting and properly estimating haze thickness.

\section{Band Extrapolation}

The employment of a blue band from a multispectral image for the HTM generation can lead to overdehazing of this band. Fig. 4 illustrates such a situation on AVNIR-2 data (see [14]). A per-band analysis should be carried out [compare Fig. 4(a) and (b)]. In order to overcome this problem, a new synthetic band is created by a linear extrapolation of two bands of the image cube:

$$
\begin{aligned}
& \operatorname{Band}_{\text {extrapol }}(x, y) \\
& \quad=\operatorname{EXTRAPOL}\left(\operatorname{Band}_{m}(x, y), \operatorname{Band}_{n}(x, y)\right)
\end{aligned}
$$

where $\operatorname{Band}_{\text {extrapol }}(x, y)$ is the extrapolated band and $\operatorname{Band}_{m}(x, y)$ and $\operatorname{Band}_{n}(x, y)$ are two bands from the data cube used for an extrapolated band creation. An employment 


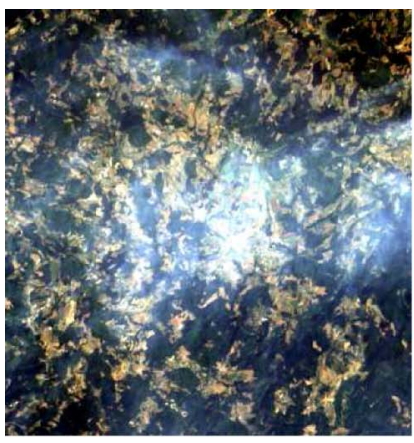

(a)

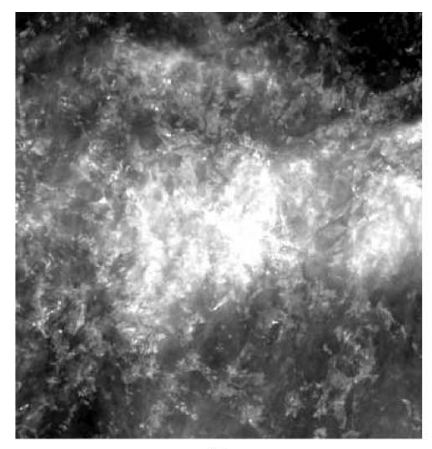

(b)

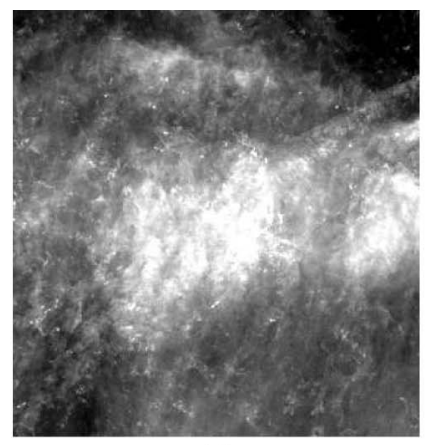

(c)

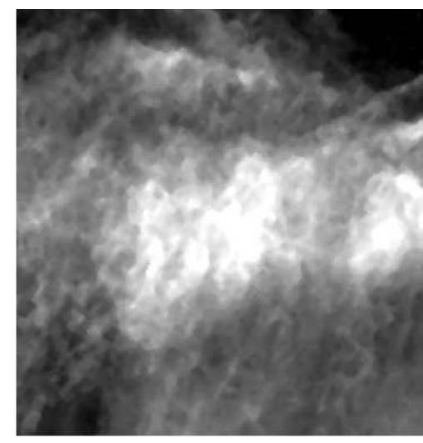

(d)

Fig. 5. Estimation of the HTM in Landsat 8 OLI data. (a) RGB true color composite (bands 4, 3, and 2). (b) Band 1 (0.4430 $\mu$ m). (c) Extrapolated band Band $_{\text {extrapol }}(\lambda=0.4233$; extrapolated using Landsat 8 OLI bands 1 and 2). (d) HTM calculated on the extrapolated band in (c).

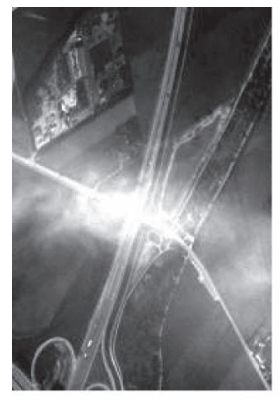

(a)

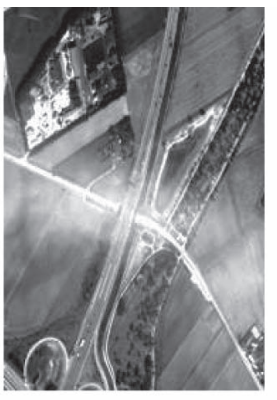

(b)

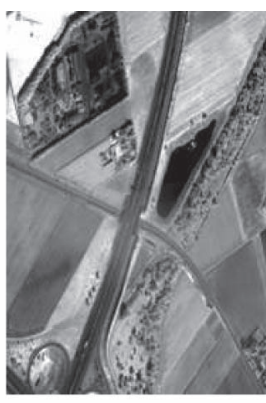

(c)

Fig. 6. Example of varying haze thickness in WorldView-2 spectral channels. (a) Band $1(0.4283 \mu \mathrm{m})$. (b) Band $4(0.6080 \mu \mathrm{m})$. (c) Band $7(0.8277 \mu \mathrm{m})$.

of the extrapolated band yields better dehazing results [see Fig. 4(a) and (c)]. Usually, the first two bands are selected (in the case of Landsat $8 \mathrm{OLI}$, Band $_{m}$ is $0.4430 \mu \mathrm{m}$, and Band $_{n}$ is $0.4825 \mu \mathrm{m}$ band). Fig. 5 illustrates an example of an extrapolated band: band 1 at $0.4430 \mu \mathrm{m}$ [Fig. 5(b)] and an extrapolated band (Fig. 5(c); $\lambda=0.4232 \mu \mathrm{m}$ ). The reflectance of the surface in the extrapolated (a lower wavelength) band is less, haze thickness is higher in comparison to the VNIR region bands, and the HTM can be estimated more precisely. In the case of noise in the interpolated band, a median filtering can be applied.

\section{Haze Thickness Per Band}

In order to dehaze a multispectral image, a subtraction of the HTM has to be done for each spectral band. The derived haze thickness is different in bands and usually decreases from the shortest to the longest wavelength band (Fig. 6). Subtraction of a constant HTM from all of the bands can lead to overdehazing in the red and near infrared bands and a loss of spectral properties in the dehazed data. To preserve spectral consistency, a band-specific HTM $\left(\mathrm{HTM}_{i}\right)$ should be calculated. Taking into account that spectral channels in the far short-wave infrared range are only marginally influenced by haze, the dehazing is performed for the visible, near infrared, and short-wave infrared bands, terminating the dehazing wavelength at $2.2 \mu \mathrm{m}$.

The thickness of the haze in each band $\left(\mathrm{HTM}_{i}\right)$ is estimated relative to the HTM calculated using the image $\operatorname{Band}_{b}(x, y)$ [see (3)]. A temporary $\operatorname{HTM~HTM}_{i}^{\prime}(x, y)$ is calculated for each band $i$, and the regression coefficient (slope of the fitted line

in linear regression) $\left(k_{i}\right)$ of $\operatorname{HTM}_{i}^{\prime}(x, y)$ versus $\operatorname{HTM}(x, y)$ is stored in an array $(\mathbf{K})$ of regression coefficients. The regression coefficient is calculated using the pixel values in the segmented hazy regions (haze mask is employed). According to haze thickness, the regression coefficients are expected to have a decreasing trend for the bands from the shortest to the longest wavelength. A check if $\mathbf{K}$ has a meaningful decreasing trend and a correction of the trend are performed. Each $k_{i+1}$ should be less than $k_{i}$ and $\left(k_{i+1}-k_{i}\right) \leq 0.1$. The initial start value of 0.1 is an empirical threshold, and it was calculated experimentally on multispectral data. It might be changed during the later iteration (7). A linear scaling of $\mathbf{K}$ into the range [1, 0] is performed [the maximal value corresponds to maximal HTM density in the first band with shortest wavelength, and the minimal value corresponds to haze thickness in the longest wavelength band at $2.2 \mu \mathrm{m}$ (haze is not visible)]. Therefore, a band-specific haze thickness is subtracted from the original data. Note on the linear scaling of $K$ : in principle, a nonlinear scaling with wavelength could also be used, requiring scenederived parameters that influence the radiative transfer, e.g., the haze particle size distribution and scattering phase functions. This information cannot usually be retrieved. However, we checked the potential influence of the phase function: in the relevant spectral region for haze removal (400-900 $\mathrm{nm})$, the change of haze scattering functions with wavelength is negligible. This behavior was checked with the MODTRAN [15] haze/fog models. Summarizing, $\mathrm{HTM}_{i}$ is calculated as follows:

$$
\operatorname{HTM}_{i}^{\prime}(x, y)=D A R K \_P I X \_S E A R C H\left(\operatorname{Band}_{i}(x, y), w\right)
$$

where the nonoverlapping window size is $w=21$

$$
k_{i}=\operatorname{SLOPE}\left(\operatorname{HTM}(x, y), \operatorname{HTM}_{i}^{\prime}(x, y)\right)
$$

where $k_{i}$ is the regression coefficient (slope of the fitted line) for band $i$ and $\mathbf{K}$ is the array of the coefficients $\left(k_{i} \in \mathbf{K}\right)$. $\operatorname{HTM}(x, y)$ is the independent variable, and $\operatorname{HTM}_{i}^{\prime}(x, y)$ is the dependent variable. Note that the $\operatorname{HTM}(x, y)$ and $\operatorname{HTM}_{i}^{\prime}(x, y)$ values are taken only in the segmented hazy regions (haze mask is employed)

$\mathbf{K}=L I N E A R \_S C A L E(\mathbf{K},[1,0])$ for $\lambda=\left[\lambda_{\text {blue }}, 2.2 \mu \mathrm{m}\right]$. 


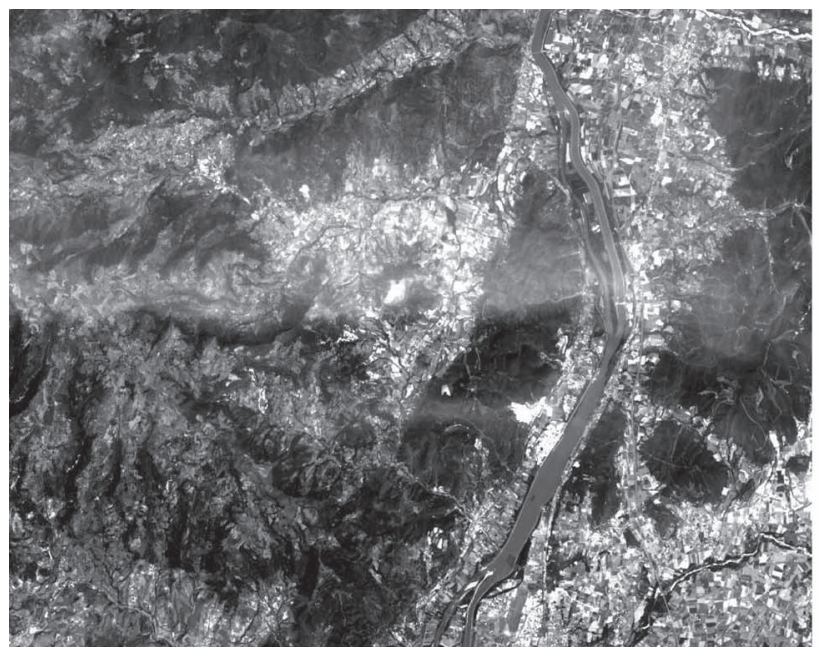

(a)

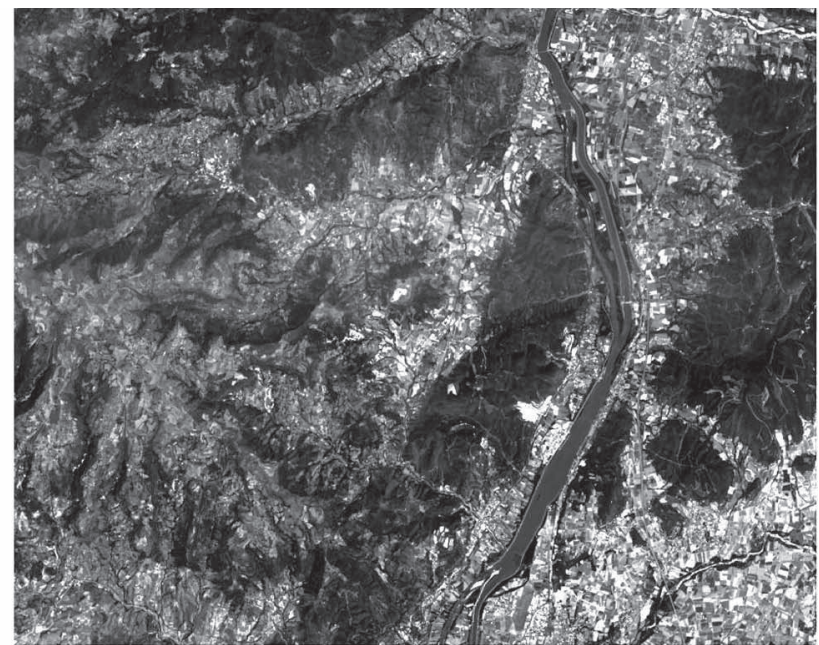

(b)

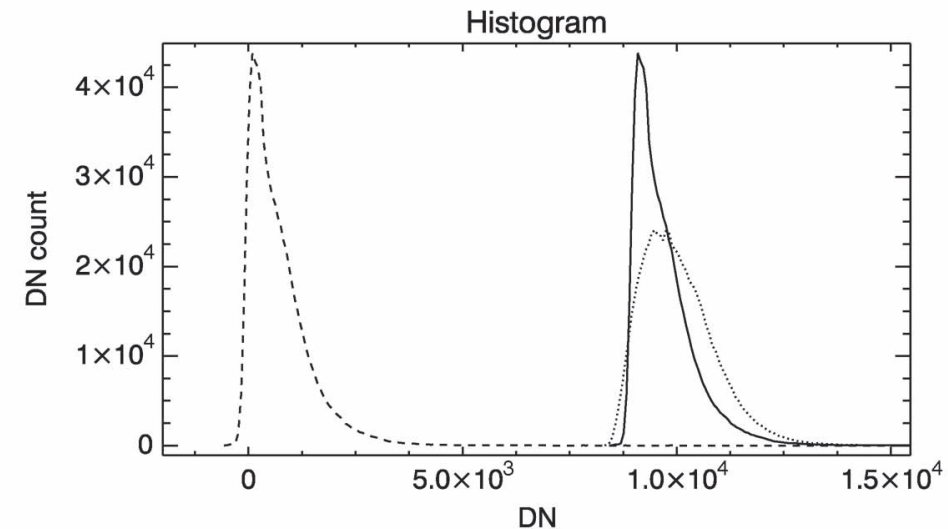

(c)

Fig. 7. Landsat 8 OLI band $3(0.525-0.600 \mu \mathrm{m})$ dehazing (subset). (a) Original band 3. (b) Dehazed band 3. (c) Histogram of the original band 3 (dotted), dehazed (dashed), and the compensated [aerosol thickness compensation (10)] band (solid line). Note that negative values can appear (regions of shadows, vegetation, deep water, and dark objects) since the HTM is calculated using the extrapolated band and the HTM has higher thickness values than the original bands.

Note: after the $K$ scaling, the initial 0.1 difference between the consecutive bands might be changed

$$
\operatorname{HTM}_{i}(x, y)=\operatorname{HTM}_{i}^{\prime}(x, y) * k_{i}, k_{i} \in \mathbf{K} .
$$

\section{E. Dehazing and Compensating of Clear Scene Aerosol}

A subtraction of $\operatorname{HTM}_{i}(x, y)$ from the band $\operatorname{DN}_{i}^{\text {sensor }}(x, y)$ should recover the dehazed band

$$
\mathrm{DN}_{i}(x, y)=\mathrm{DN}_{i}^{\text {sensor }}(x, y)-\operatorname{HTM}_{i}(x, y) .
$$

The subtraction removes the HTM (haze plus clear scene aerosol) thickness, and the data should be compensated for the clear scene aerosol fraction. The HTM pixels outside the haze contain values representing the clear scene aerosol thickness (the aerosol thickness is also calculated in haze-free areas). A compensation for the aerosol thickness is performed (see example in Fig. 7)

$$
\begin{array}{r}
\mathrm{DN}_{i}^{\prime}=\mathrm{DN}_{i}+A B S\left(M E A N \left(\mathrm{DN}_{i}^{\text {sensor }}[\text { non_haze })\right.\right. \\
\left.-M E A N\left(\mathrm{DN}_{i}[\text { non_haze }]\right)\right) .
\end{array}
$$

The huge difference between the histograms of the original and dehazed bands can be explained as follows. Landsat 8 OLI data have a high dynamic range, and the lowest DN value in band 3 (Fig. 7) is higher than 6000. Therefore, the darkest objects in the band have a minimum value equal or higher than 6000 . The subtraction of the generated HTM leads to such high difference between the original and dehazed band histograms. The dehazing on the data produced by a sensor with a low radiometry (e.g., Landsat 7, 8-b data) will produce a much smaller difference between the histograms.

\section{F. Method Pseudocode}

The method for multispectral image dehazing can be summarized into a pseudocode for easy implementation:

\section{begin}

* x_size, y_size, b_size are the dimensions of the multispectral data cube.

* Load multispectral data cube Band $_{i}, i=\left[1, b \_s i z e\right]$.

* Apply mean-shift segmentation to the band in the red spectral region.

* Threshold the segmented image (threshold value is equal to the mean). Save result into bright_obj. 
TABLE I

Parameters of the Landsat 8 Oli Data Sets: Hazy and Haze Free (Reference)

\begin{tabular}{|l|l|l|}
\hline & Hazy & Haze free (reference) \\
\hline \hline Landsat scene ID & LC81960292013195LGN00 & LC81970292013186LGN00 \\
\hline WRS path & 196 & 197 \\
\hline WRS row & 29 & 29 \\
\hline Acquisition date and time (UTC) & 14 July 2013, 10:25:28 & 7 July 2013, 10:31:39 \\
\hline Solar zenith angle & $28.0^{\circ}$ & $26.9^{\circ}$ \\
\hline Solar azimuth angle & $138.0^{\circ}$ & $137.3^{\circ}$ \\
\hline Sensor tilt angle & $0.0^{\circ}$ & $0.0^{\circ}$ \\
\hline
\end{tabular}

* Create a new synthetic band by extrapolation

$$
\begin{aligned}
& \text { Band }_{\text {extrapol }}=\left(\text { Band }_{0.4430 \mu \mathrm{m}}+\left(\text { Band }_{0.4430 \mu \mathrm{m}}\right.\right. \\
&\left.\left.-0.95 * \text { Band }_{0.4825 \mu \mathrm{m}}\right)\right)>0.0
\end{aligned}
$$

* Calculate haze thickness map:

for $x:=w / 2$ to $x \_$size step $w$ do

for $y:=w / 2$ to $y \_$size $\operatorname{step} w$ do

$\operatorname{HTM}(x, y)$

$=\operatorname{MIN}\left(\right.$ Band $\left._{\text {extrapol }}(x-w / 2: x+w / 2, y-w / 2: y+w / 2)\right)$

\section{od}

od

* Resize the HTM to the size $\mathrm{x} \_$size, $\mathrm{y} \_$size.

* Calculate haze mask $(w=21)$ :

for $x:=w / 2$ to $x \_$size $\operatorname{step} w$ do

for $y:=w / 2$ to $y \_s i z e$ step $w$ do

$$
\begin{aligned}
& \operatorname{HTM}_{\text {haze_mask }}(x, y) \\
& =\operatorname{MIN}\left(\operatorname{Band}_{\text {extrapol }}(x-w / 2: x+w / 2, y-w / 2: y+w / 2)\right)
\end{aligned}
$$

od

od

* Resize the HTM $\mathrm{M}_{\text {haze_mask }}$ to the size $\mathrm{x} \_$size, $\mathrm{y} \_$size.

* Threshold the HTM $\mathrm{HTaze}_{\text {mask }}$ by the MEAN $\left(\mathrm{HTM}_{\text {haze_mask }}\right)$, create haze_pixels and non_haze_pixels lists.

* Save haze (haze_pixels) and nonhaze (non_haze_pixels) pixel lists.

* Calculate array $k_{i} \in \mathbf{K}, i=\left[1, b \_s i z e\right]$ of regression coefficients $(w=3)$ :

for $i:=1$ to $b \_s i z e$ step 1 do

for $x:=w / 2$ to $x \_$size step $w$ do

for $y:=w / 2$ to $y \_s i z e$ step $w$ do

$\operatorname{HTM}_{i}(x, y)$

$=\operatorname{MIN}\left(\operatorname{Band}_{i}(x-w / 2: x+w / 2, y-w / 2: y+w / 2)\right)$

od

od

$k_{i}=S L O P E_{-} C O E F F$

$$
\times\left(\operatorname{HTM}[\text { haze_pixels }], \mathrm{HTM}_{i}[\text { haze_pixels }]\right)
$$

od

* Linear scaling of the array $\mathbf{K}$ :

$$
\mathbf{K}=\operatorname{LINEAR\_ SCALE}(\mathbf{K},[1,0])
$$

* Perform triangular interpolation of the HTM pixels corresponding to mask bright_obj.

\section{$*$ Perform dehazing:}

for $i:=1$ to $b \_$size step 1 do

$$
\begin{aligned}
& \text { Band }_{i}^{\prime}=\text { Band }_{i}-\operatorname{HTM} * k_{i}, k_{i} \in \mathbf{K} . \\
& \operatorname{Band}_{i}^{\prime \prime}=\text { Band }_{i}^{\prime}+\operatorname{ABS}\left(\text { MEAN } \left(\text { Band }_{i}[\text { non_haze })\right.\right. \\
& \text { - MEAN (Band } \left.\left.d_{i}^{\prime}[\text { non_haze }]\right)\right) \text {. }
\end{aligned}
$$

$$
\begin{gathered}
\text { od } \\
\text { end }
\end{gathered}
$$

\section{Product Evaluation And Discussion}

\section{A. Landsat 8 OLI Dehazing and Evaluation With Haze-Free Data}

A quantitative evaluation of a dehazing method is a difficult task since the appropriate hazy data together with ground truth are difficult to collect. Since a strict validation is not possible, a more realistic approach is chosen: a pair of hazy and hazefree images was found. The images have minimal time difference and minimal difference of the Sun/sensor geometry. The minimal difference of the image acquisition conditions allows us to use the haze-free image as a reference and to perform an evaluation of the dehazing method by a comparison of the spectral information.

Landsat 8 OLI hazy and haze-free data were analyzed to make an evaluation of the dehazed product. A pair of scenes for one region (Montelimar, France) was selected for this purpose. The data were acquired with a time difference of 7 days and in the same acquisition geometry conditions. The detailed information on the data is given in Table I. Taking into account a negligible difference in the acquisition geometry and a small time shift, the haze-free image can be employed as a reference for haze-free data. An evaluation of the dehazing method can be performed by a comparison of the dehazed and reference image spectra.

Fig. 8 illustrates the dehazing results for a Landsat 8 OLI subscene. Visual interpretation of the dehazed image [Fig. 8(b)] and comparison to the reference [Fig. 8(c)] per band and using a true color RGB composition confirm the conclusion that the dehazing method performs well. The calculated coefficient array is $\mathbf{K}=[1.0,0.9010,0.8164,0.8863,0.7813,0.4022,0.1]$ for the channels $0.4430,0.4825,0.5625,0.6650,0.8700$, 1.6100 , and $2.2000 \mu \mathrm{m}$, respectively, without the cirrus band B9 (at $1.38 \mu \mathrm{m})$.

Fig. 9 illustrates top of atmosphere (TOA) reflectance spectra collected in the hazy, dehazed, and reference images. Fig. 9(a) illustrates that the spectra collected in the haze-free regions have the same shape. The plus symbol (hazy image) and the asterisk symbol (dehazed image) closely agree, illustrating that the dehazing of data outside the haze regions does not modify 


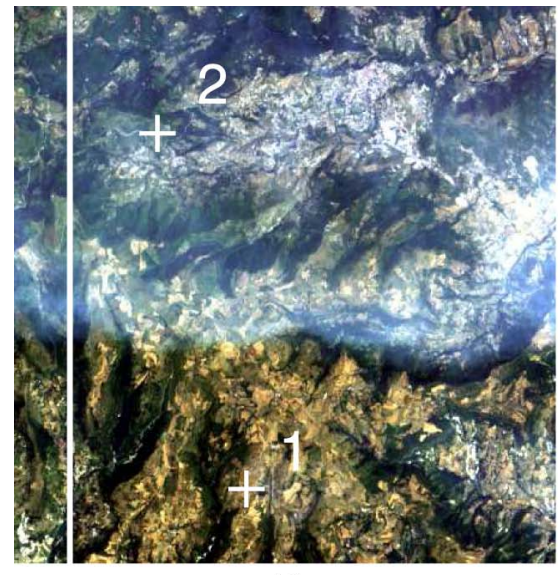

(a)

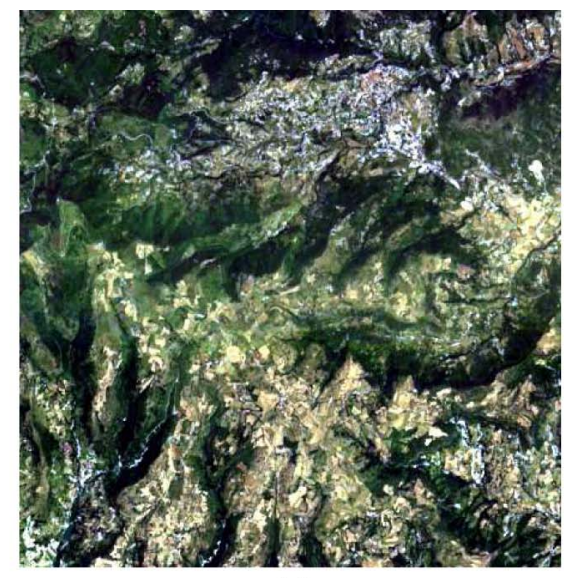

(b)

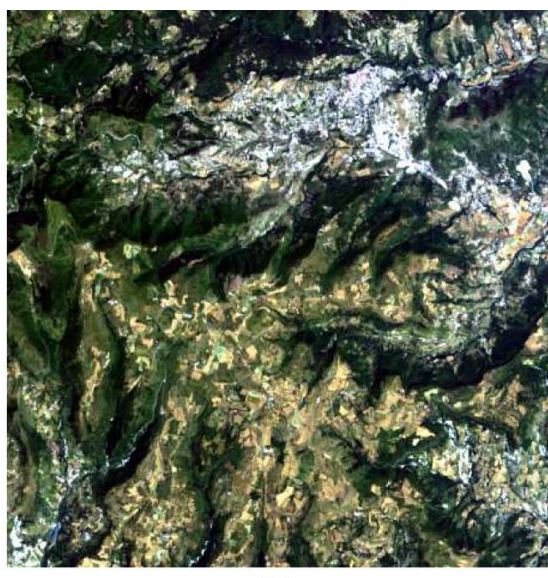

(c)

Fig. 8. Dehazing of Landsat 8 OLI data (subset). (a) Hazy data RGB true color band combination (July 14, 2013; scene ID: LC81960292013195LGN00). (b) Dehazed data RGB band combination. (c) Haze-free reference data collected on another day (July 7, 2013; scene ID: LC81970292013186LGN00).

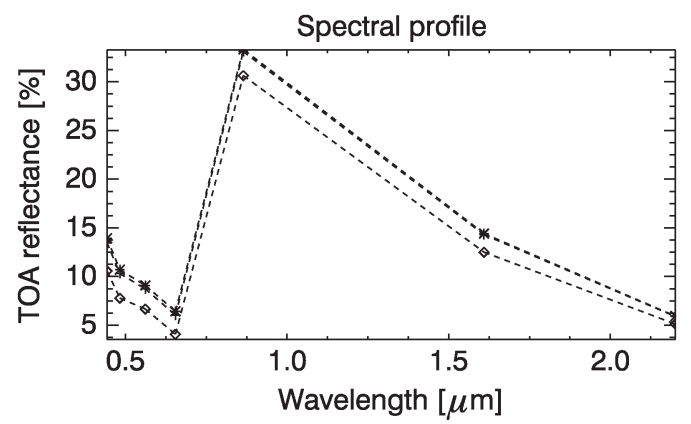

(a)

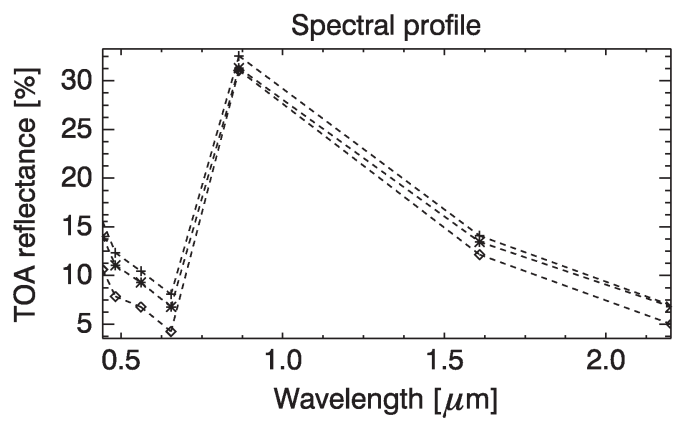

(b)

Fig. 9. Spectra collected $(3 \times 3$ window average) in Landsat 8 OLI haze-free [cross 1; Fig. 8(a)] and hazy [cross 2; Fig. 8(a)] regions for the original hazy (plus symbol), dehazed (asterisk symbol), and reference (diamond symbol) TOA reflectance images. (a) Haze-free spectra (the original and dehazed image spectra are very close). (b) Spectra taken in the hazy region.

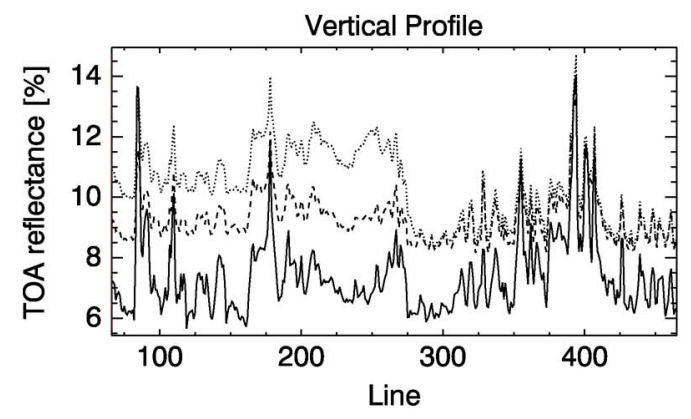

(a)

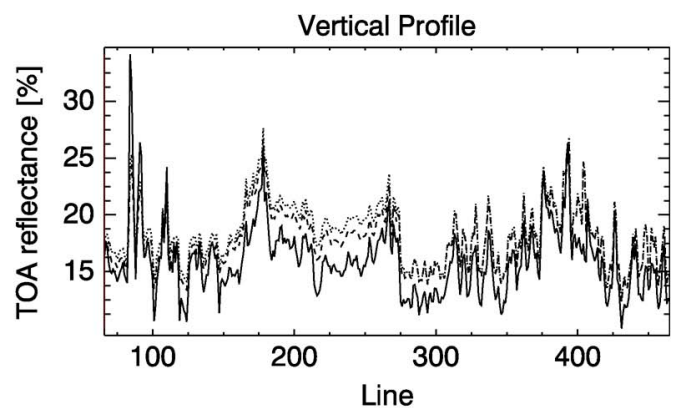

(b)

Fig. 10. Band profiles (Fig. 8(a), vertical white line) taken from the original hazy (dotted line), dehazed (dashed line), and reference (solid line) Landsat 8 OLI images. (a) Band $3(0.5613 \mu \mathrm{m})$. (b) Band $6(1.6100 \mu \mathrm{m})$. The difference in the hazy region between the two profile lines clearly illustrates that the dehazing was performed, and the shape of the profile after dehazing shows a preservation of the scene structure (left part of the profiles). The profiles have almost identical shapes in the haze-free region (right part of the profiles).

the spectral properties of the original data. These two spectra have the same shape as the reference image spectrum (diamond symbol). Fig. 9(b) presents the spectra collected in a hazy region in the hazy (plus symbol), dehazed (asterisk symbol), and reference (diamond symbol) images. The spectra collected from the dehazed image have the same shape as the spectrum collected in the reference image. Thus, the method produces spectrally consistent dehazed data. The difference between the dehazed and reference spectra can be attributed to residual scene coregistration errors, different atmospheric conditions, and residual haze thickness errors.
A comparison of the channel intensity profiles in the hazy and dehazed image is another verification. Fig. 10 presents band $3(0.5613 \mu \mathrm{m})$ and band $6(1.6091 \mu \mathrm{m}$; TOA reflectance $)$ profiles for the hazy (dotted), dehazed (dashed), and reference (solid line) images. In the haze-free region (right part of the graph in Fig. 10), the original and dehazed profiles have similar shapes. In the hazy region (left part of the graph), the dehazed profile illustrates a noticeable dehazing enhancement of the band intensities together with a preservation of the surface structure by comparing the dotted (hazy band) and solid (hazefree reference band) lines. Again, the remaining differences 


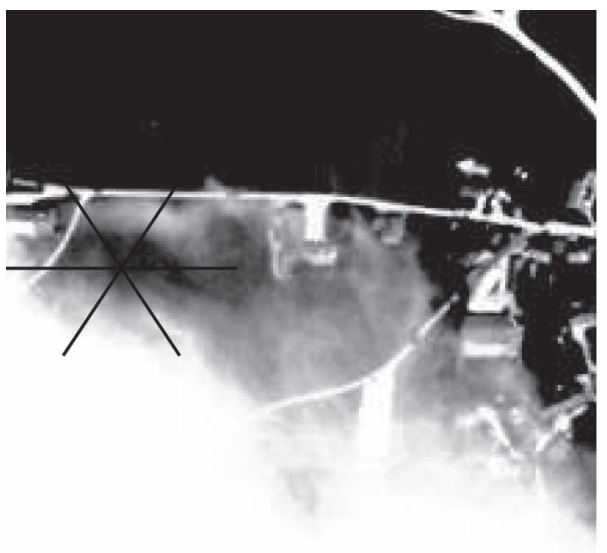

(a)

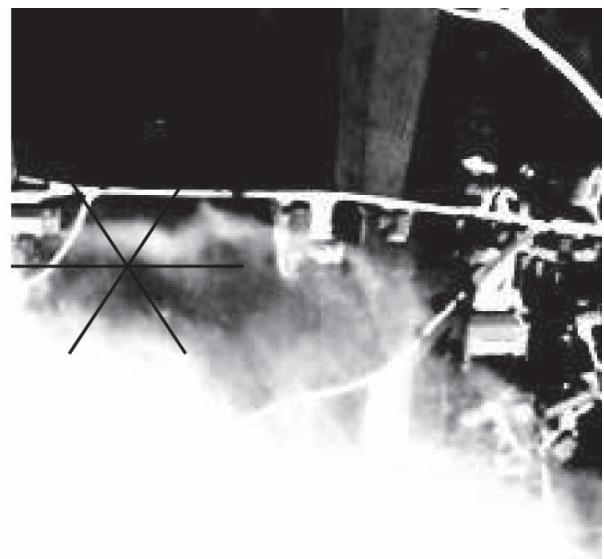

(b)

Fig. 11. Parallax effect in WorldView-2 multispectral data leads to haze shift in the bands of multispectral image. A vertical shift of the haze is noticeable between (a) band $1(0.4283 \mu \mathrm{m})$ and (b) band $2(0.4791 \mu \mathrm{m}$; the star has the same position on both images).

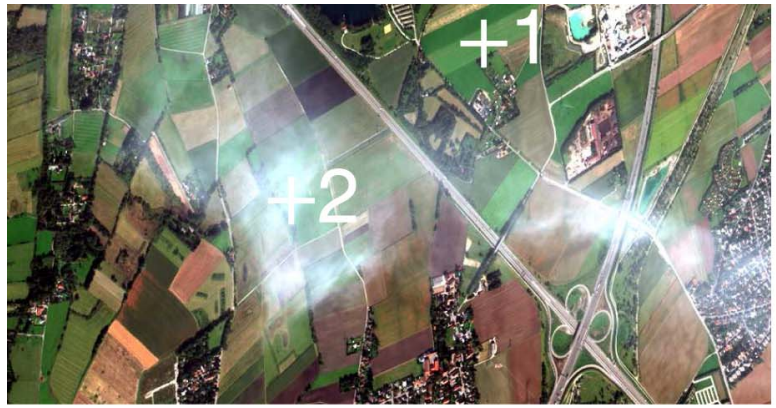

(a)

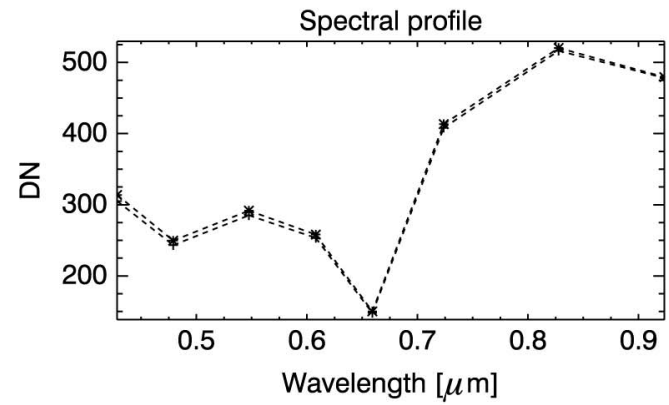

(c)

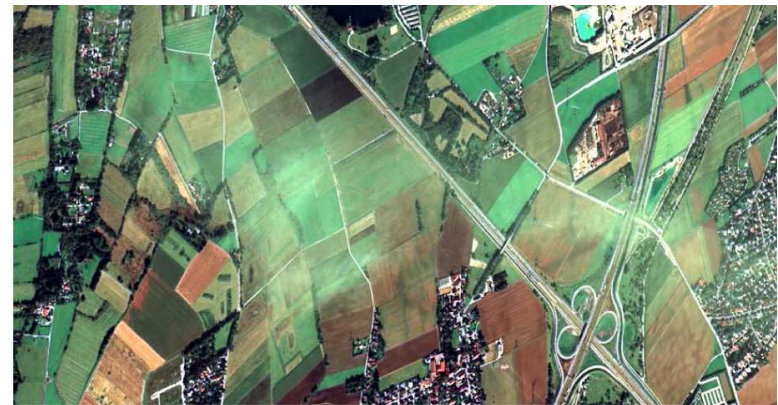

(b)

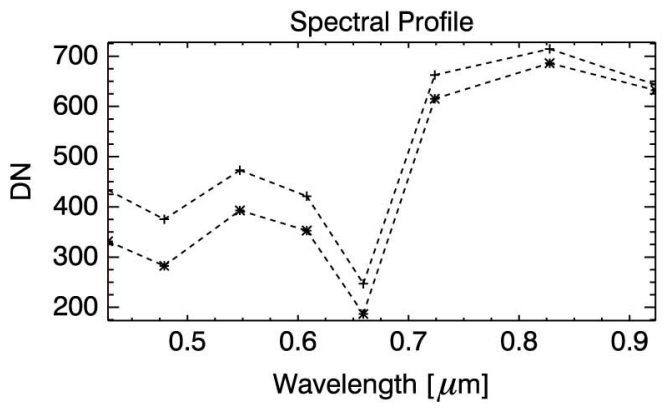

(d)

Fig. 12. Dehazing of WorldView-2 data (subset). (a) Hazy data RGB true color band combination. (b) Dehazed data RGB band combination. (c) Spectral profiles of the hazy (plus symbol) and dehazed (asterisk symbol) images outside the haze (cross 1). (d) Spectral profiles of the hazy (plus symbol) and dehazed (asterisk symbol) images inside the haze (cross 2).

are likely due to different atmospheric conditions and residual haze.

The execution time on a Landsat 8 OLI subset of size $2647 \times$ 5035 pixels ( 7 bands) is approximately 1 min on Intel Core 2 Duo, using an IDL implementation [16], [17] of the presented algorithm.

\section{B. WorldView-2}

Dehazing of WorldView-2 multispectral data has one important aspect: due to the parallax effect, the position of the haze in bands $1,4,6$, and 8 is different from bands $2,3,5$, and 7 . The markers in Fig. 11(a) and (b) illustrate a vertical shift of the haze in the scene (band 1 and band 2, respectively). The employment of band 1 and band 2 does not allow us to extrapolate a new band with a proper haze thickness because the HTM will be calculated with parallax errors (haze borders and thickness will not be precise). The employment of such an HTM does not allow an accurate haze removal. To overcome this problem, it is necessary to calculate two HTMs: HTM 1 for bands 1, 4, 6, and 8 and $\mathrm{HTM}_{2}$ for bands 2, 3, 5, and 7. Bands 1 and 4 are used for extrapolation to create a new band and further the calculation of $\operatorname{HTM}_{1}(\lambda=0.4131)$ for bands 1, 4, 6, and 8. Bands 2 and 3 are used for extrapolation to create a new band and further the calculation of $\operatorname{HTM}_{2}(\lambda=0.4131)$ for bands 2, 3, 5, and 7. Then, $\mathrm{HTM}_{1}$ is linearly stretched to $\mathrm{HTM}_{2}$ using a local sliding window (the size is $10 \times 10$ pixels) to match the HTMs.

A dehazing example for WorldView-2 data is presented in Fig. 12. The eight-channel data were acquired on September 23, 2010, over the city of Munich at 11:15:44.08 UTC. The 


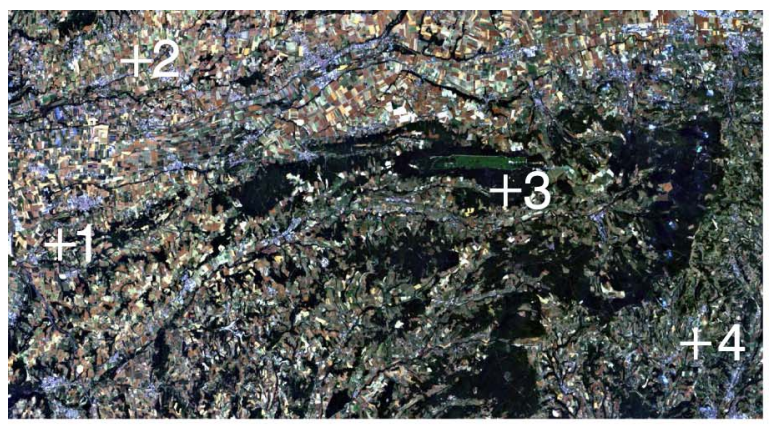

(a)

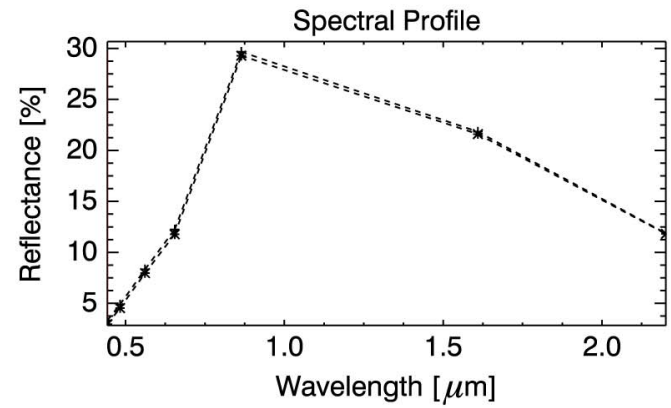

(c)

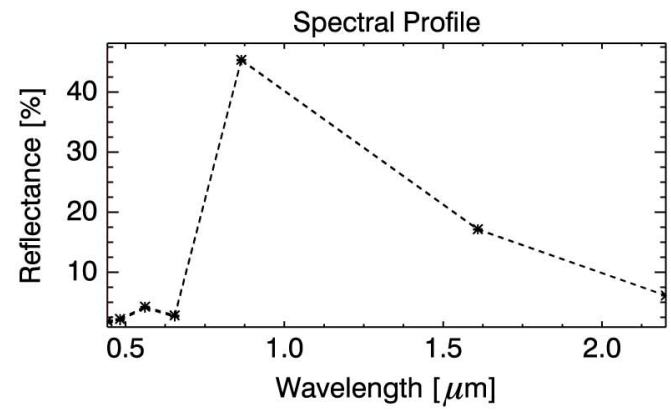

(e)

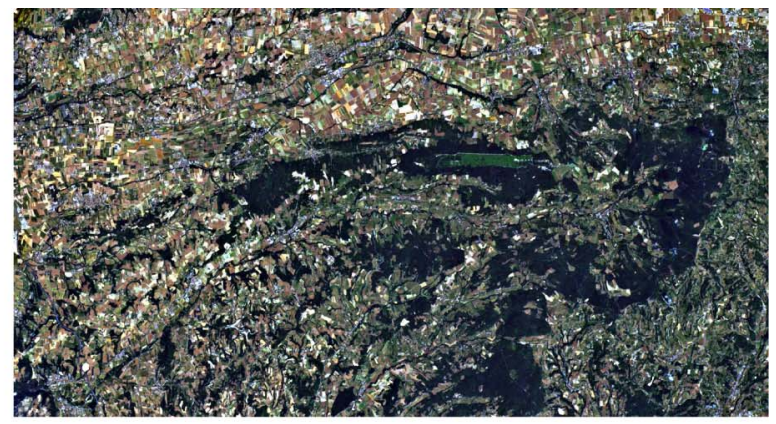

(b)

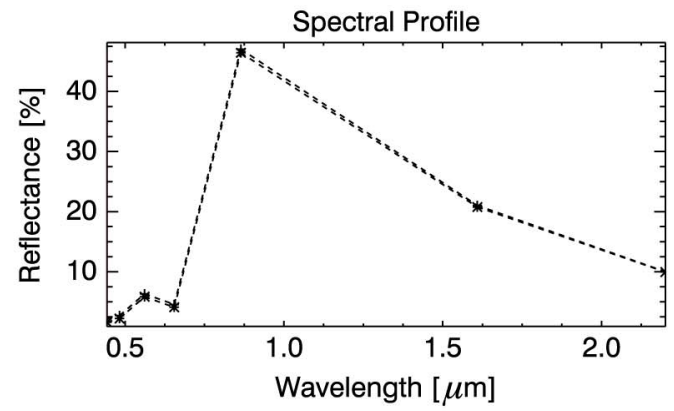

(d)

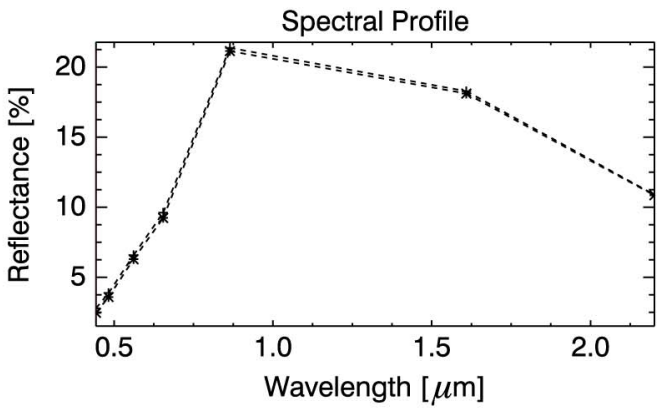

(f)

Fig. 13. Atmospheric correction (ATCOR-3) of haze-free Landsat 8 OLI and the same data after dehazing. (a) Haze-free RGB true color band combination. (b) Dehazed data RGB band combination. (c)-(f) Spectral profiles of the haze-free (plus symbol) and the atmospherically corrected (asterisk symbol) for crosses $1-4$, respectively.

calculated coefficient array is $\mathbf{K}=[1.0,0.9077,0.7832,0.6733$, $0.5802,0.4628,0.2739,0.1]$. Since a different haze thickness is subtracted from the bands, the dehazed spectra have the same shape but with a slightly higher difference in the first four bands [Fig. 12(d)] because these are more influenced by haze.

The execution time on a WorldView-2 subset of size $2525 \times$ 1919 pixels ( 8 bands; note that a calculation of two HTMs is required, and $\mathrm{HTM}_{1}$ is locally stretched to $\mathrm{HTM}_{2}$ ) is approximately 1 min on Intel Core 2 Duo, using an IDL implementation of the algorithm. The main part of the dehazing time is required for the HTM calculation (in case of WorldView-2, two HTMs are necessary to calculate), e.g., being 2.4 times smaller, the WorldView-2 cube requires nearly the same time for the dehazing as the Landsat 8 OLI data cube, respectively.

\section{Atmospheric Correction of Dehazed Data}

The dehazing method also has to properly operate on hazefree multispectral data. Radiometric distortions should not be introduced into the data, and the atmospheric correction of both the original (haze-free) and haze-free data after dehazing should result in a good agreement of the surface reflection spectra. A comparison of spectra taken in the original (hazefree) and dehazed images allows us to demonstrate that the dehazing method does not influence the radiometric properties of the data.

An example is given in Fig. 13: A haze-free subscene of the original Landsat 8 OLI (see Section III-A) was processed with our method, and both the original and dehazed images are run for atmospheric correction in the ATCOR-3 software [8]. The calculated mean visibilities (dense dark vegetation based method) for the original (haze-free) and dehazed subscenes are the following: 19.9 and $20.6 \mathrm{~km}$, respectively. Fig. 13 presents the original haze-free [Fig. 13(a)] and dehazed [Fig. 13(b)] images. The plots in Fig. 13(c)-(f) demonstrate that the collected spectra in the images have a negligible difference, leading to the conclusion that the developed dehazing method does not distort the radiometrical properties if applied to clear scenes.

\section{CONCLUSION}

A new multispectral data dehazing method has been developed. The algorithm removes spatially varying haze based on the HTM. An evaluation of the dehazing method using 
a clear and a hazy scene from the same area demonstrates that the dehazing results are spectrally consistent. Spectrally consistent dehazing is also achieved on other multi- and hyperspectral data such as AVNIR, Landsat 7, RapidEye, and AVIRIS. The method can be applied to the original data DN counts or the calibrated radiance data. It is fully automatic and can be employed as a chain in batch processing systems of remotely sensed data. The dehazed data can further be used for atmospheric/topographic correction. A limitation of the method is the following: in rare cases of scenes with absolutely flat and highly reflective surfaces (desert and snow), it could be impossible to locate dark objects and to estimate an HTM. However, such scenes are usually not very common and not of practical interest. Future improvements of the method will deal with possible border effects caused by the triangulation and haze removal for hyperspectral imagery.

\section{ACKNOWLEDGMENT}

The authors would like to thank European Space Imaging (EUSI) for the collection and provision of Digitalglobe WorldView-2 data.

\section{REFERENCES}

[1] R. Richter, "Atmospheric correction of satellite data with haze removal including a haze/clear transition region," Comput. Geosci., vol. 22, no. 6, pp. 675-681, Jul. 1996.

[2] G. D. Moro and L. Halounova, "Haze removal for high-resolution satellite data: A case study," Int. J. Remote Sens., vol. 28, no. 10, pp. 2187-2205, May 2007.

[3] Y. Zhang and B. Guindon, "Quantitative assessment of a haze suppression methodology for satellite imagery: Effect on land cover classification performance," IEEE Trans. Geosci. Remote Sens., vol. 41, no. 5, pp. 10821089, May 2003.

[4] C. Liu, J. Hu, Y. Lin, S. Wu, and W. Huang, "Haze detection, perfection and removal for high spatial resolution satellite imagery," Int. J. Remote Sens., vol. 32, no. 23, pp. 8685-8697, 2011.

[5] K. He, J. Sun, and X. Tang, "Single image haze removal using dark channel prior," IEEE Trans. Pattern Anal. Mach. Intell., vol. 33, no. 12, pp. 2341-2353, Dec. 2011.

[6] P. Chavez, "An improved dark-object subtraction technique for atmospheric scattering correction of multispectral data," Remote Sens. Environ., vol. 24, no. 3, pp. 459-479, Apr. 1988.

[7] P. Chavez, "Image-based atmospheric corrections-Revisited and improved," Photogramm. Eng. Remote Sens., vol. 62, no. 9, pp. 1025-1036, Sep. 1996.

[8] ATCOR, Atmospheric/Topographic Correction for Satellite Imagery. ReSe Applications Schläpfer, Langeggweg 3, CH-9500 Wil, Switzerland. [Online]. Available: www.rese.ch

[9] B. Waske and S. van der Linden, "Classifying multilevel imagery from SAR and optical sensors by decision fusion," IEEE Trans. Geosci. Remote Sens., vol. 46, no. 5, pp. 1457-1466, 2008.

[10] B. Waske and J. Benediktsson, "Fusion of support vector machines for classification of multisensor data," IEEE Trans. Geosci. Remote Sens., vol. 45, no. 12, pp. 3858-3866, Dec. 2007.

[11] F. Pacifici, F. Del Frate, W. Emery, P. Gamba, and J. Chanussot, "Urban mapping using coarse SAR and optical data: Outcome of the 2007 GRSS data fusion contest," IEEE Geosci. Remote Sens. Lett., vol. 5, no. 3, pp. 331-335, Jul. 2008.

[12] N. Longbotham, F. Pacifici, T. Glenn, A. Zare, M. Volpi, D. Tuia, E. Christophe, J. Michel, J. Inglada, J. Chanussot, and Q. Du, "Multimodal change detection, application to the detection of flooded areas: Outcome of the 2009-2010 data fusion contest," IEEE J. Sel. Topics Appl. Earth Observ. Remote Sens., vol. 5, no. 1, pp. 331-342, Feb. 2012.

[13] D. Comaniciu and P. Meer, "Mean shift: A robust approach toward feature space analysis," IEEE Trans. Pattern Anal. Mach. Intell., vol. 24, no. 5, pp. 603-619, May 2002.

[14] P. Schwind, M. Schneider, G. Palubinskas, T. Storch, R. Muller, and R. Richter, "Processors for ALOS optical data: Deconvolution, DEM generation, orthorectification, atmospheric correction," IEEE Trans. Geosci. Remote Sens., vol. 47, no. 12, pp. 4074-4082, Dec. 2009.
[15] A. Berk, G. Anderson, P. Acharya, and E. Shettle, MODTRAN 5.2.0.0 User's Manual. Hanscom AFB, MA, USA: Air Force Res. Lab., 2008, pp. 01731-3010.

[16] D. W. Fanning, IDL Programming Techniques, 2nd ed. Fort Collins, CO, USA: Fanning Software Consulting, 2000

[17] Exelis visual information solutions. [Online]. Available: www.excelisvis com

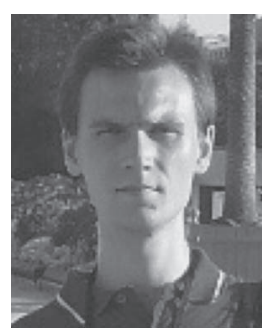

Aliaksei Makarau received the Dipl.-Ing. degree in computer science from Belarusian State University of Informatics and Radioelectronics, Minsk, Belarus, in 2003 and the Ph.D. degree in technical sciences from the United Institute of Informatics Problems, Minsk, in 2008.

His dissertation was on fast methods for multispectral image fusion and processing. He is currently with the Department of "Photogrammetry and Image Analysis," Remote Sensing Technology Institute (IMF), German Aerospace Centre (DLR). His research interests include atmospheric correction, multisensory data fusion, and pattern recognition.

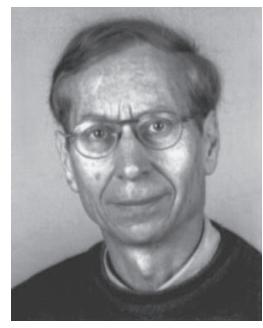

Rudolf Richter received the M.Sc. degree in physics from the Technical University of Munich, Munich, Germany, in 1973 and the Ph.D. (Dr.-Ing.) degree in engineering from the Technical University of Dresden, Dresden, Germany, in 1991

$\mathrm{He}$ is a DLR Senior Scientist conducting concept development, modeling, and simulation of airborne/ spaceborne hyperspectral instruments. It involves advanced techniques and interaction with scientists associated with theory and remote sensing experiments. He developed the ATCOR model, one of the standard codes for atmospheric and topographic correction of multi- $/$ hyperspectral imagery used at universities and research laboratories. His current work focuses on the design of fully automatic processing chains for the evaluation of remotely sensed optical data from the visible to the thermal spectral region.

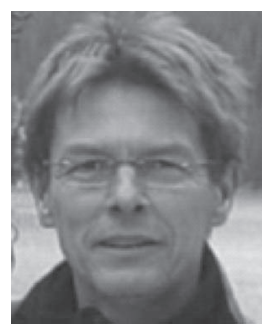

Rupert Müller received the Dipl.-Phys. degree from the Ludwig Maximilians University of Munich, Munich, Germany, in 1985.

$\mathrm{He}$ is currently a Team Leader of the "Processors and Traffic Monitoring" Group, Remote Sensing Technology Institute, German Aerospace Center (DLR), Wessling, Germany, and is responsible for the Environmental Mapping and Analysis Program processing, calibration, and validation part within the ground segment as well as several European Space Agency projects like "Prototype Processor for ALOS Optical Data." His main research interests include photogrammetric evaluation of spaceborne satellite data and digital image processing.

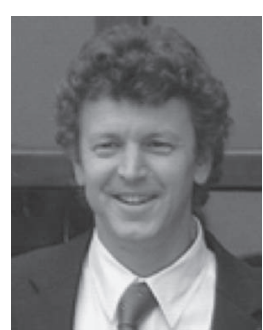

Peter Reinartz received the Dipl.-Phys. degree in theoretical physics from the University of Munich, Munich, Germany, in 1983 and the Ph.D. (Dr.-Ing) degree in civil engineering from the University of Hannover, Hanover, Germany, in 1989.

His dissertation was on statistical optimization of classification methods for multispectral image data. $\mathrm{He}$ is the Head of the Department of "Photogrammetry and Image Analysis," Remote Sensing Technology Institute (IMF), German Aerospace Centre (DLR), and is a Professor of geoinformatics with the University of Osnabrueck, Osnabrueck, Germany. He has more than 20 years of experience in image processing and remote sensing and over 150 publications in these fields. His main interests are direct georeferencing, stereo photogrammetry and data fusion, generation of digital elevation models, and interpretation of VHR image data from sensors like WorldView, GeoEye, etc. $\mathrm{He}$ is also engaged in using remote sensing data for disaster management and using high-frequency time series of airborne image data for real-time application in case of disasters as well as for traffic monitoring. 